\title{
Stevens-Johnson Syndrome Following Treatment With Carbamazepine for a Mood Disorder
}

\author{
Albert Coleman M.D., M.P.H. \\ Psychiatry Residency Program, Department of Postgraduate Education, Yale University, New Haven, CT \\ B. Trappler M.D. \\ Kings County Hospital Center Department of Psychiatry, Brooklyn, NY
}

Follow this and additional works at: https://jdc.jefferson.edu/jeffjpsychiatry

Part of the Psychiatry Commons

Let us know how access to this document benefits you

\section{Recommended Citation}

Coleman, Albert M.D., M.P.H. and Trappler, B. M.D. (1996) "Stevens-Johnson Syndrome Following

Treatment With Carbamazepine for a Mood Disorder," Jefferson Journal of Psychiatry. Vol. 13 : Iss. 1 , Article 8.

DOI: https://doi.org/10.29046/JJP.013.1.007

Available at: https://jdc.jefferson.edu/jeffjpsychiatry/vol13/iss1/8

This Article is brought to you for free and open access by the Jefferson Digital Commons. The Jefferson Digital Commons is a service of Thomas Jefferson University's Center for Teaching and Learning (CTL). The Commons is a showcase for Jefferson books and journals, peer-reviewed scholarly publications, unique historical collections from the University archives, and teaching tools. The Jefferson Digital Commons allows researchers and interested readers anywhere in the world to learn about and keep up to date with Jefferson scholarship. This article has been accepted for inclusion in Jefferson Journal of Psychiatry by an authorized administrator of the Jefferson Digital Commons. For more information, please contact: JeffersonDigitalCommons@jefferson.edu. 


\title{
Stevens-Johnson Syndrome Following Treatment with Carbamazepine for a Mood Disorder
}

\author{
Albert Coleman, M.D., M.P.H., ${ }^{1}$ \\ B. Trappler, M.D. ${ }^{2}$
}

\begin{abstract}
A case of Stevens-Johnson Syndrome (SJS) induced by carbamazepine in a psychiatric patient with schizo-affective disorder is reported. The patient also had diabetes mellitus and was on glyburide which posed an initial dilemma at the onset of SJS as to the causative drug. In view of the expanding use of carbamazepine in psychiatric patients and the potentially lethal consequences of SJS, the authors suggest a risk management approach when initiating Carbamazepine therapy to reduce the incidence and morbidity of the syndrome.
\end{abstract}

Among the adverse dermatologic manifestations associated with drug therapy, Stevens-Johnson Syndrome (SJS) stands out as a severe, potentially lethal adverse effect. SJS has been associated with the use of carbamazepine and also phenobarbital and phenytoin, mostly in neurological cases. It is of interest to note that in the case of carbamazepine, the first such described case occurred in the year it was introduced (1). The overall incidence from studies of carbamazepine-induced SJS is low (an average of about 5 per $1,000,000$ cases) and is considered uncommon $(2,3,4)$. Carbamazepine is an iminodibenzyl derivative with a structure similar to the tricyclic antidepressants. In clinical neurology, carbamazepine has been effective in the treatment of psychomotor and grand mal seizures, as well as trigeminal neuralgia.

SJS is a clinical syndrome presumed to be a hypersensitivity reaction manifested initially with prodromal symptoms of fever, malaise and a sore throat. The prodromal phase is then followed in some days (up to 14 days) by an acute polymorphous dermatologic syndrome manifested as erythematous maculo-papular like lesions, target lesions, bullae, vesicles, involvement of at least two mucous membranes, conjunctivitis and an associated systemic toxic state.

Since the Japanese (5) postulated and described the possible effectiveness of carbamazepine in affective disorders and the subsequent confirmation of this in later controlled clinical trials (6), carbamazepine has been increasingly used by psychia-

\footnotetext{
'Psychiatry Residency Program, Department of Postgraduate Education, Yale University, Grace Building, 61 1, 25 Park Street, New Haven, CT 06504.

${ }^{2}$ Kings County Hospital Center, Department of Psychiatry (G-53), (S.U.N.Y.-H.S.C. Box 1203), 450 Clarkson, Brooklyn, NY 11203.
} 
trists as a mood stabilizer. Along with the increasing use of carbamazepine in contemporary psychiatric practice is the rare occurrence of SJS as a side effect. However, very few cases have been described in the psychiatric literature so far (7). We present a case of carbamazepine-associated SJS in a psychiatric patient who also had diabetes mellitus and was also on treatment with glyburide in our institution.

\section{CASE REPORT}

A thirty-five year old caucasian male, single, domiciled, unemployed, college educated with a greater than 15 years psychiatric history and multiple past psychiatric admissions, treated in the past with haloperidol and lithium was admitted to our institution on February 6, 1994, with a long diagnosis of schizoaffective disorder. On admission, along with paranoid and grandiose delusions, the patient also had a pronounced manic state. He was started on haloperidol, lithium and benztropine. Since he also suffered from adult onset diabetes mellitus (diagnosed in another institution a year ago prior to this admission), and was on treatment with regular insulin as an outpatient, this was continued.

On admission, the patient had fluctuating mildly increased blood sugar levels, for which the unit internist prescribed glyburide while the patient was weaned off insulin. Glyburide was started eight days after admission. From the past history the patient was not compliant with lithium therapy and this was also confirmed upon admission when he refused to take lithium. He subsequently reported that he was non-compliant on lithium because of a fixed idea that his sister was collaborating with his therapist to label him a bipolar in order to disqualify him from a family inheritance.

As the patient's manic syndrome continued, an alternative mood stabilizer was sought. Carbamazepine was found acceptable to the patient as a substitute for lithium ten days after admission. Carbamazepine was begun and titrated upwards. Ten days after initiation of carbamazepine therapy, the patient developed flu-like symptoms with a sore throat. Two days later, he developed dermatologic lesions consisting principally of an erythematous maculpapular rash. Glyburide was discontinued as this reaction was thought to have been caused by the medication. A sliding scale regular insulin coverage regimen was instituted in place of glyburide, while the patient remained on haloperidol and carbamazepine. In addition, oral prednisone $40 \mathrm{mg}$ was added to the treatment.

Three days after the appearance of the flu-like symptoms and dermatologic rash, the dermatology consultant diagnosed drug-induced morbilliform eruption secondary to glyburide. The dermatologist recommended continued treatment with oral prednisone, while adding triamcinolone for dermal application. However, the patient's dermal eruption got worse, and he developed bleeding intranasal lesions, ulcerating buccal lesions, increased malaise, and generalized bodily aches and discomfort. Hematological and blood chemistry work up results were within normal limits. Two days from the first dermatologic consult, a suspicion of possible SJS was raised and a second dermatology consult was sought. At this time, the patient was febrile $(100 \mathrm{~F})$ and because of the oral lesions had difficulty eating. Review of the case by the senior dermatologist led to diagnosis of SJS attributed to carbamazepine. (Two days before the initial dermal eruption, the serum carbamazepine level was $8.4 \mu \mathrm{g} / \mathrm{ml}$; two days after the eruption serum carbamazepine level measured $9.3 \mu \mathrm{g} / \mathrm{ml}$ ). Carbamazepine was discontinued immediately and because of the worsening physical condition of the patient, a decision was made to transfer the patient to the medical floor.

Treatment on the medical floor consisted of haloperidol, oral prednisone and a sliding 
scale insulin coverage regimen. Seven days from the beginning of the flu-like symptoms, the intermittent nasal bleeding subsided, the feeling of malaise was reduced, and oral lesions started showing signs of remission. The patient's manic symptoms, along with the delusions, had now almost resolved. Patient was discharged home from the medicine unit thirty-two days after his initial psychiatric admission with plans for follow up in the outpatient psychiatry and medicine clinics. A suggestion was also made for a trial with valproic acid as a mood stabilizer.

\section{DISCUSSION AND COMMENTARY}

A small number of psychotropics have been associated with SJS, frequently cited are phenobarbital, phenytoin, fluoxitine, meprobamate and carbamazepine $(8,9)$. In the psychiatry literature, carbamazepine-associated SJS has been rare. The first such described case was reported in the United States (10) the most recent case was reported from Canada (11).

Considering the potentially lethal consequences of SJS and the increasing use of carbamazepine in psychiatry (not only as a mood stabilizer, but also for other psychiatric indications including impulse control and alcohol detoxification) (12), continued use of carbamazepine should be considered against the background of current therapeutic information. Some authors have suggested an increased risk of SJS when neuroleptics are combined with carbamazepine (13). Others have recommended patch testing prospective recipients for hypersensitivity to the drug (14). This should, however, be done having in mind the risk of a false positive test result and an occasional unpleasant dermatologic reaction to the patch (15). Other authors have postulated a possible desensitization procedure for carbamazepine hypersensitivity reactions (16). The idea of desensitizing carbamazepine-induced SJS patients seems plausible, but considering the sometimes fulminant and lethal course of this syndrome, the outcome in these patients might carry a far bigger risk. Considering the relative benefit and expanded use of carbamazepine in psychiatry, psychiatrists need to assess the clinical use of this drug on a case by case basis with an awareness of the associated adverse reactions.

This particular case was unique considering the fact that the patient was also a diabetic on glyburide. Initially, at the manifestations of the prodromal signs this posed a problem of diagnosis, considering that glyburide is a sulfonylurea compound. Although some cases of SJS have been associated with use of some sulphur-containing drugs $(17,18)$, a thorough search failed to demonstrate a case or citation for SJS induced by glyburide.

In this case, even after the glyburide was discontinued and the patient was maintained on carbamazepine, prednisone and haloperidol, the clinical picture worsened until the carbamazepine was discontinued. This makes a plausible case for considering carbamazepine as the SJS inducer. This case, to date, is the second psychiatric case of carbamazepine-induced SJS in a patient in the United States since the original was published in 1987 (7).

Finally, to reduce the incidence of carbamazepine-induced SJS in psychiatric practice, we suggest a risk assessment profile approach (as used in preventive 
medicine practice) when carbamazepine therapy is being considered. This entails consideration of and application of all information as pertains to carbamazepine hypersensitivity, possible association of the syndrome in neuroleptic carbamazepine combinations, consideration for using other known alternative to carbamazepine with similar desired effects (for example, valproic acid). In the end, when a decision is made to proceed with carbamazepine therapy, a more proactive approach of inquiring from patients about symptoms/signs associated with the prodromal stage of the syndrome should be sought. In the same light, the patient should be instructed and taught to look out for symptoms and signs and immediately inform the staff to facilitate early intervention and secondary prevention of this syndrome.

\section{REFERENCES}

1. Coombes BW: Stevens-Johnson Syndrome associated with Carbamazepine (Tegretol), Medical Journal of Australia 1965; 1:895-896.

2. Strom BI, Carson JL, Halpern AC, et al: A population-based study of Stevens-Johnson Syndrome. Incidence and antecedent drug exposures. Archives of Dermatology 1991; 127(6):831-8.

3. Strom BL, Carson JL, Halpern AC, et al: Using a claims database to investigate drug-induced Stevens-Johnson Syndrome. Statistics in Medicine 1991; 10(4):565-76.

4. Schopf E, Stuhmer A, Rzany B, et al: Toxic epidermal necrolysis and Stevens-Johnson Syndrome. An epidermiologic study from West Germany. Archives of Dermatology 1991; 127(6):839-42.

5. Tekezaki H, Hanaoka M: The Use of Carbamazepine (Tegretol) in the Control of Manic-Depressive Psychosis and other Manic Depressive States, Clinical Psychiatry 1971; 13:173-183.

6. Ballenger James C, Post Robert M.: Carbamazepine in Manic Depressive Illness. A New Treatment, AMJ of Psychiatry 1981, July, 137:782-790.

7. Powers MJ \& Co. (Publishers): Carbamazepine and Stevens-Johnson Syndrome. In Psychiatry Drug Alerts. 1994 April, Vol. VIII, Number 4.

8. Pelekanos J, Camfield P, Gordon K: Allergic rash due to antiepileptic drugs, clinical features and management. Epilepsy 1991; 31:554-559.

9. Bryant BG, Matthews BL, Stevens-Johnson syndrome. Drug Intelligence and Clinical Pharmacy 1986; 20:489-493.

10. Fawcett R: Erythema multiforme major in a patient with carbamazepine. Journal of Clinical Psychiatry 1987; 48:416-417.

11. Pagliaro L, Pagliaro A: Carbamazepine-induced Stevens-Johnson syndrome. Hospital and Community Psychiatry 1993; October, 44:999-1000.

12. Arana GW, Hyman SE: Psychiatric uses of anticonvulsants. In Handbook of Psychiatric Drug Therapy. Little Brown 108-127.

13. Wong KE: Stevens-Johnson Syndrome in neuroleptic-carbamazepine combination. Singapore Medical Journal 1990; October, 31(5):432-433.

14. Rodriguez Mosquera M, Engelsias A, Saez A, Vidual C: Patch test diagnosis of Carbamazepine sensitivity. Contact Dermatitis 1991; August, 25(2):137-138.

15. Vaillant L, Camenen I, Lorett: Patch testing with carbamazepine: Reinduction of an exfoliate dermatitis. Archives of Dermatology 1989, 125:299. 
16. Hermle L, Spitzer M: Erfolgreiche hyposensitization ungsebehandlumg bei einum patienten mit einer schizoaffektiven Psychose and carbamazepin allergie successful hyposensitization treatment of a patient with schizo-affective psychosis and carbamazepione allergy (translation from German) Nervenarzt, 1993; March, 64(3):208-210.

17. Bamba MG, Elder AT, Gray JA, Minns RA: Fatal Stevens-Johnson Syndrome associated with fansidar and chloroquine, Journal of Infection 1986; July, 13(1):13.

18. Navin TR, Miller KD, Satriale RF, Lobel HO: Adverse reactions associated with pyrimethamine-sulfadoine prophylaxis for pneumocystis carinii infections in AIDS (letter) Lancet 1985; June 8, 1(8441): 132 . 